\title{
A GENERALIZATION OF NORMAL CONGRUENCES OF CIRCLES *
}

BY J. L. WALSH

1. Introduction. A congruence of circles in three-dimensional space is said to be normal if every circle of the congruence is normal to three surfaces. Normal congruences have long been studied, $\dagger$ and one of their principal properties is expressed in the theorem that if a variable circle $C$ is normal to the three fixed surfaces $S_{1}, S_{2}, S_{3}$ at the points $P_{1}, P_{2}, P_{3}$ respectively, and if the point $P_{4}$ is determined by a real constant cross ratio with $P_{1}, P_{2}, P_{3}$, then as $C$ varies the point $P_{4}$ traces a surface which is also orthogonal to $C$.

It is the purpose of the present note to consider a type of congruence to which we shall give the name of isogonal congruence and which is a generalization of the notion of normal congruence. A congruence of circles is said to be isogonal if every circle of the congruence cuts three surfaces at equal angles in such a way that when the circle is inverted into a straight line $L$, the tangent planes to the corresponding surfaces at their points of intersection with $L$ are all parallel. That is, every sphere through a circle of the congruence cuts at equal angles the three surfaces at their points of intersection with that circle. It is to be noted that the term isogonal might well be given to a still larger type of congruence of circles, but in the present paper the term will be used only in the restricted sense indicated.

We shall prove (Theorem III) that if a congruence is isogonal there are not merely three surfaces but a one-parameter family of surfaces which have the isogonal property, and all the surfaces of the family can be obtained as in the case of normal congruences.

* Presented to the Society, December 27, 1922.

$\dagger$ By Ribaucour, Darboux, Bianchi, Eisenhart, and Coolidge, among others. Detailed references are given by Coolidge, $A$ Treatise on the Circle and the Sphere, Oxford, 1916, Chap. XV. 
Isogonal congruences of circles are thus a generalization of normal congruences of circles, of normal congruences of lines, and of certain congruences of lines which naturally arise in connection with the parallel mapping of surfaces*. Isogonal congruences are particularly interesting because in general three arbitrary surfaces determine one or several such congruences isogonal to them. If one fixed circle $C$ is isogonal to three surfaces, there is in general one and only one congruence of circles isogonal to those surfaces and containing $C$. For the condition of isogonality for a circle $C^{\prime}$ and three surfaces $C_{1}, C_{2}, C_{3}$ is satisfied if two spheres through the circle $C^{\prime}$ cut at equal angles the surfaces $C_{1}, C_{2}, C_{3}$ at their points of intersection with $C^{\prime}$. This is equivalent to four independent conditions on all the six-parameter family of circles in space.

Let us proceed to investigate the analogue in the plane of the isogonal congruence.

2. Isogonal Series in the Plane. The name isogonal series shall be given to a one-parameter family of circles in the plane such that each circle of the family cuts at equal angles three curves, and in such a way that when the circle is inverted into a straight line, the tangents to the transformed curves at their points of intersection with the transformed circle are all parallel. In general, three arbitrary curves determine one or more isogonal series, and if a circle $C$ cuts isogonally three curves there is in general one and only one isogonal series which contains $C$ and all of whose circles cut isogonally those three curves. For the condition of isogonality to three eurves involves two independent conditions on the three-parameter family of all circles of the plane. We shall proceed to prove the following theorem.

Theorem I. Let a variable circle $C$ cut three fixed curves $C_{1}$, $C_{2}, C_{3}$ isogonally at the variable points $z_{1}, z_{2}, z_{3}$ respectively. Then the point $z_{4}$ defined by the real constant cross ratio

$$
\lambda=\left(z_{1}, z_{2}, z_{3}, z_{4}\right) \equiv \frac{\left(z_{1}-z_{2}\right)\left(z_{3}-z_{4}\right)}{\left(z_{2}-z_{3}\right)\left(z_{4}-z_{1}\right)}
$$

* Isogonal congruences are also a generalization of a type of congruences of circles considered by F. W. Beal, Annals of Mathematics, (2), vol. 17 (1915-16), pp. 180-170. 
traces a curve $C_{4}$ such that the circle $C$ cuts isogonally $C_{1}, C_{2}$, $C_{3}, C_{4}$ at the points $z_{1}, z_{2}, z_{3}, z_{4}$.

We fix our attention on a particular circle $C$ and its points of intersection $z_{1}{ }^{\prime}, z_{2}{ }^{\prime}, z_{3}{ }^{\prime}$ (supposed distinct) with $C_{1}, C_{2}, C_{3}$. We shall prove that if $z_{1}, z_{2}, z_{3}$ move simultaneously from $z_{1}{ }^{\prime}, z_{2}{ }^{\prime}, z_{3}{ }^{\prime}$ along $C_{1}, C_{2}, C_{3}$ in any way whatever, then the point $z_{4}$ defined by (1) traces a curve which $C$ cuts isogonally with $C_{1}, C_{2}, C_{3}$.

If $z_{1}$ moves from $z_{1}^{\prime}$ along $C_{1}$, but $z_{2}$ and $z_{3}$ are kept coincident with $z_{2}^{\prime}$ and $z_{3}{ }^{\prime}$, then $z_{4}$ moves from $z_{4}{ }^{\prime}$ along a curve which is cut by $C$ isogonally with $C_{1}$. This becomes obvious if $z_{3}^{\prime}$ is transformed to infinity; equation (1) then represents a transformation $\left(z_{1}, z_{4}\right)$ of similitude with $z_{2}^{\prime}$ as center, while $C$ is a straight line which is unchanged by the transformation. Likewise if $z_{2}$ moves from $z_{2}^{\prime}$ along $C_{2}$, but $z_{1}$ and $z_{3}$ are kept coincident with $z_{1}^{\prime}$ and $z_{3}{ }^{\prime}$, then $z_{4}$ moves from $z_{4}{ }^{\prime}$ along a curve which is cut by $C$ isogonally with $C_{2}$. A similar fact obtains if $z_{3}$ moves from $z_{3}{ }^{\prime}$ along $C_{3}$. Thus, independent infinitesimal changes of $z_{1}, z_{2}, z_{3}$ from $z_{1}{ }^{\prime}, z_{2}{ }^{\prime}, z_{3}{ }^{\prime}$ along $C_{1}{ }^{\prime}$, $C_{2}{ }^{\prime}, C_{3}{ }^{\prime}$ move $z_{4}$ from $z_{4}{ }^{\prime}$ along a curve of the kind described, so simultaneous infinitesimal changes of $z_{1}, z_{2}, z_{3}$ from $z_{1}{ }^{\prime}, z_{2}{ }^{\prime}$, $z_{3}{ }^{\prime}$ along $C_{1}{ }^{\prime}, C_{2}{ }^{\prime}, C_{3}{ }^{\prime}$ also move $z_{4}$ from $z_{4}{ }^{\prime}$ along a curve $C_{4}$ such that $C$ cuts the curves $C_{1}, C_{2}, C_{3}, C_{4}$ işogonally at $z_{1}{ }^{\prime}$, $z_{2}{ }^{\prime}, z_{3}{ }^{\prime}, z_{4}{ }^{\prime}{ }^{*}$ 'This completes the proof of Theorem I.

Theorem $\mathrm{I}$ is particularly interesting in the case that $C_{1}$,

* The detailed analysis of the differentials involved is extremely simple in the present case. We have by differentiation of (1) and substitution of (1) in the result,

(1') $\quad d z_{4}=\frac{\left(z_{1}-z_{2}\right) d z_{3}+\left(z_{3}-z_{4}\right)\left(d z_{1}-d z_{2}\right)-\lambda\left(z_{2}-z_{3}\right) d z_{1}-\lambda\left(z_{4}-z_{1}\right)\left(d z_{2}-d z_{3}\right)}{\left(z_{1}-z_{2}\right)+\lambda\left(z_{2}-z_{3}\right)}$.

Transform the circle $C$ of Theorem I into the axis of reals, identify the real values $z_{i}{ }^{\prime}$ of Theorem I with the $z_{i}$ of $\left(1^{\prime}\right)$, and identify the variables $z_{i}$ of Theorem I with the $z_{i}+d z_{i}$ of $\left(1^{\prime}\right)$. All the quantities in $\left(1^{\prime}\right)$ except the differentials are real; all the differentials of the right-hand member have the same argument $(\bmod \pi)$, so $d z_{4}$ has also that same argument $(\bmod \pi)$.

Thus if $z_{1}(t), z_{2}(t), z_{3}(t), z_{4}(t)$ are solutions of a Riccati equation whose cross ratio is a real constant, then whenever the $z_{i}(t)$ vary as functions of $t$, they trace paths that are cut isogonally by the circle on which the $z_{i}(t)$ lie. 
$C_{2}, C_{3}$ are all circles. In this case we have the following theorem.*

Theorem II. Let $C_{1}, C_{2}, C_{3}$ be three fixed non-coaxial circles. Then the circles $C$ which cut isogonally $C_{1}, C_{2}, C_{3}$ at points $z_{1}, z_{2}, z_{3}$ form four distinct series, $\dagger$ each of which is composed of the circles of a coaxial family. If there are considered the circles. $C$ of but one isogonal series, the point $z_{4}$ defined by the real constant cross ratio

$$
\lambda=\left(z_{1}, z_{2}, z_{3}, z_{4}\right)
$$

traces a circle $C_{4}$ which is such that $C$ cuts isogonally $C_{1}, C_{2}, C_{3}$, $C_{4}$ at $z_{1}, z_{2}, z_{3}, z_{4}$.

If $C_{1}, C_{2}, C_{3}$ are coaxial but not all tangent at a single point, there is but one series of circles $C$ cutting them isogonally, namely the circles of the coaxial family conjugate to the family to which $C_{1}, C_{2}, C_{3}$ belong, and all these circles $C$ cut the three given circles orthogonally. But the points $z_{1}, z_{2}, z_{3}$ may be chosen on $C$ and on the circles $C_{1}, C_{2}, C_{3}$ in four essentially different ways, always so that $C$ cuts $C_{1}, C_{2}, C_{3}$ isogonally at $z_{1}, z_{2}, z_{3}$. Thus we still have four circles $C_{4}$ and for any particular choice of $C_{4}$, the circle $C$ cuts isogonally $C_{1}, C_{2}, C_{3}, C_{4}$ at $z_{1}, z_{2}, z_{3}, z_{4}$.

If $C_{1}, C_{2}, C_{3}$ are all tangent at a single point $P$, any circle $C$ through $P$ cuts the original circles isogonally at the intersections of $C$ and those circles distinct from $P$, so no isogonal series is defined. We can still obtain the four circles $C_{4}$, however, by requiring respectively (1) that no point $z_{1}, z_{2}, z_{3}$ shall coincide with $P$; $(2)$ that $z_{1}$ shall always lie at $P$; $C$ must then be orthogonal to $C_{1}, C_{2}, C_{3}$; (3), (4) similarly for $z_{2}$ and $z_{3}$. Always the circle $C_{4}$ is traced by the point $z_{4}$, and the circle $C$ cuts $C_{1}, C_{2}, C_{3}, C_{4}$ isogonally at $z_{1}, z_{2}, z_{3}, z_{4}$.

3. Isogonal Congruences in Space. Theorem I and its proof

* See Walsh, Transactions of this Society, vol. 22 (1921), pp. 101116; Lemma IV.

† By a proper convention for the angle between two circles, these four systems are described respectively by saying that $C$ cuts $C_{1}, C_{2}, C_{3}$ all at the same angle or one of those circles at an angle supplementary to the angle cut on the other two. A similar remark obtains below for Theorem IV. 
as just given extend directly to space. Let us prove the following theorem.

Theorem III. Let a variable circle $C$ cut isogonally three fixed surfaces $C_{1}, C_{2}, C_{3}$ at the variable points $P_{1}, P_{2}, P_{3}$. Then the point $P_{4}$ defined by the real constant cross ratio

$$
\lambda=\left(P_{1}, P_{2}, P_{3}, P_{4}\right)
$$

traces a surface $C_{4}$ such that the circle $C$ cuts isogonally $C_{1}, C_{2}$, $C_{3}, C_{4}$ at the points $P_{1}, P_{2}, P_{3}, P_{4}$.

We fix our attention on a particular circle $C$ and its points of intersection $P_{1}{ }^{\prime}, P_{2}{ }^{\prime}, P_{3}{ }^{\prime}$ (supposed distinct) with $C_{1}, C_{2}, C_{3}$. We shall prove that as $P_{1}, P_{2}, P_{3}$ move from $P_{1}{ }^{\prime}, P_{2}{ }^{\prime}, P_{3}{ }^{\prime}$ on $C_{1}, C_{2}, C_{3}$ in any way whatever, then $P_{4}$ as defined by (2) traces a surface $C_{4}{ }^{\prime}$ such that $C$ cuts isogonally $C_{1}, C_{2}, C_{3}, C_{4}{ }^{\prime}$ at $P_{1}{ }^{\prime}$, $P_{2}{ }^{\prime}, P_{3}{ }^{\prime}, P_{4}{ }^{\prime}$, where $P_{4}{ }^{\prime}$ is defined by $\lambda=\left(P_{1}{ }^{\prime}, P_{2}{ }^{\prime}, P_{3}{ }^{\prime}, P_{4}{ }^{\prime}\right)$.

If $P_{1}$ moves from $P_{1}^{\prime}$ along $C_{1}$, but $P_{2}$ and $P_{3}$ are kept coincident with $P_{2}{ }^{\prime}$ and $P_{3}{ }^{\prime}$, then $P_{4}$ traces a surface which is cut by $C$ at $P_{4}{ }^{\prime}$ isogonally with $C_{1}, C_{2}, C_{3}$ at $P_{1}{ }^{\prime}, P_{2}{ }^{\prime}, P_{3}{ }^{\prime}$. The corresponding fact holds if $P_{2}$ or $P_{3}$ is allowed to move on $C_{2}$ or $C_{3}$ while the other two of the original three points are kept fixed. Independent infinitesimal changes of $P_{1}, P_{2}, P_{3}$ from $P_{1}{ }^{\prime}, P_{2}{ }^{\prime}, P_{3}{ }^{\prime}$ along $C_{1}, C_{2}, C_{3}$ therefore move $P_{4}$ along a surface of the kind described, so simultaneous infinitesimal changes of these points must move $P_{4}$ along a surface $C_{4}{ }^{\prime}$ such that $C$ cuts isogonally $C_{1}, C_{2}, C_{3}, C_{4}{ }^{\prime}$ at $P_{1}{ }^{\prime}, P_{2}{ }^{\prime}, P_{3}{ }^{\prime}, P_{4}{ }^{\prime}$. Thus even if a congruence is not isogonal but a single circle $C$ of the congruence cuts isogonally the surfaces $C_{1}, C_{2}, C_{3}$, then $C$ cuts isogonally with $C_{1}, C_{2}, C_{3}$ the surface traced by the point $P_{4}$ defined by (2).

We leave to the reader the proof of the following theorem, which is the space analogue of Theorem II.

Theorem IV. Let $C_{1}, C_{2}, C_{3}$ be three fixed non-coaxial spheres. Then the circles $C$ which cut isogonally $C_{1}, C_{2}, C_{3}$ at points $P_{1}, P_{2}, P_{3}$ form four distinct congruences, each of which is composed of the circles through two points, real, coincident, or imaginary. If there are considered the circles $C$ of but one isogonal congruence, the point $P_{4}$ defined by the real constant 
cross ratio

$$
\lambda=\left(P_{1}, P_{2}, P_{3}, P_{4}\right)
$$

traces a sphere $C_{4}$ which is such that $C$ cuts isogonally $C_{1}, C_{2}$, $C_{3}, C_{4}$ at $P_{1}, P_{2}, P_{3}, P_{4}$.

If the spheres $C_{1}, C_{2}, C_{3}$ are coaxial but not all tangent at a single point, there is but one congruence of circles cutting them isogonally; all of these circles $C$ cut $C_{1}, C_{2}, C_{3}$ orthogonally. However, the points $P_{1}, P_{2}, P_{3}$ may be chosen on $C$ and on their proper spheres in four essentially different ways, and in each case $C$ cuts isogonally $C_{1}, C_{2}, C_{3}$ at $P_{1}, P_{2}$, $P_{3}$. Thus the point $P_{4}$ still traces four spheres $C_{4}$; and for any particular $C_{4}$, the circle $C$ cuts isogonally $C_{1}, C_{2}, C_{3}, C_{4}$ at $P_{1}, P_{2}, P_{3}, P_{4}$.

If the spheres $C_{1}, C_{2}, C_{3}$ are all tangent at a single point $P$, any circle $C$ through $P$ cuts those spheres isogonally at the intersections of $C$ and these spheres distinct from $P$, so we have no unique isogonal congruence. There are, however, four spheres $C_{4}$ which can be obtained by requiring respectively that $P_{1}, P_{2}, P_{3}$, or that none of those points should coincide with $P$. In the former cases the circle $C$ must be orthogonal to $C_{1}, C_{2}, C_{3}$ to have the proper isogonal property; in the latter case all the circles $C$ to be considered form a complex instead of a congruence. In every case the point $P_{4}$ traces a sphere $C_{4}$ such that $C_{1}, C_{2}, C_{3}, C_{4}$ are cut isogonally by $C$ at $P_{1}, P_{2}, P_{3}, P_{4}$.

Theorems III and IV can be extended readily to any number of dimensions.

We add the remark that isogonal congruences arise in space naturally if we consider the problem of finding the locus of a point $P_{4}$ defined by the real constant cross ratio

$$
\lambda=\left(P_{1}, P_{2}, P_{3}, P_{4}\right)
$$

when the points $P_{1}, P_{2}, P_{3}$ have as their respective loci the regions $R_{1}, R_{2}, R_{3}$, or the surfaces $S_{1}, S_{2}, S_{3}$. The surfaces $C_{1}, C_{2}, C_{3}$ defining the isogonal congruence are the boundaries of the regions $R_{1}, R_{2}, R_{3}$ or the surfaces $S_{1}, S_{2}, S_{3}$ themselves. The boundary of the locus of $P_{4}$ is traced by $P_{4}$ as defined by (3) when the circle $C$ of the congruence cuts $C_{1}, C_{2}, C_{3}$ isog- 
onally at $P_{1}, P_{2}, P_{3}$. Detailed consideration of the corresponding fact for the plane has been given in a paper by the writer,* and can easily be extended to space by the reader.

HaRVARD UNIVERSITY

\section{A CORRECTION}

BY EINAR HILLE

In the June number of this Bulletin (vol. 28, No. 5, p. 261), the author published a paper with the title Convex distribution of the zeros of Sturm-Liouville functions. Through an oversight the last paragraph of the paper is inaccurate. We list the necessary corrections below.

Page 264, lines 4-10: Instead of "Note the lineal . . . $\varphi_{0}$.", read "On $l$ we mark the eventual points $a_{n}$ as well as the points where either $\arg G(z)=\arg G\left(z_{1}\right)+\pi$ or $\theta_{z} \equiv \varphi_{0}$ $(\bmod \pi)$. Let $z_{2}=z_{2}\left(\varphi_{0}\right)$ be the first of these points, different from $z_{1}$, which we encounter when proceeding along the ray, the rest of which we leave out."

Page 264, line 13: Instead of "an analytic curve", read "either of two analytic curves, namely $A\left(z_{1}\right)$ which is the locus $\arg G(z)=\arg G\left(z_{1}\right)+\pi$, and".

Page 264, lines 24-27: Instead of " $l\left(\varphi_{1}\right)$. . respectively", read " $l\left(\varphi_{1}\right)$, considered as a double ray if necessary, from $z_{2}^{-}$to $z_{2}$ and from $z_{2}$ to $z_{2}{ }^{+}$, we make the boundary curve continuous at $\varphi=\varphi_{1}$ ".

Page 264, line 28: Instead of "cuts", read "straight lines".

Page 264, line 30: After "the part of", insert " $A\left(z_{1}\right)$ and".

Page 265, first line: Leave out "on the cuts".

Same page, lines 6-10: Replace "Then ... depends upon $z_{3}$ " by "Then we can find an angle $\vartheta$ such that the two inequalities

$$
\left\{\begin{array}{rl}
\vartheta & <\theta<\vartheta+\pi ; \\
2 k \pi & <\theta<2(k+1) \pi ;
\end{array} \quad \theta=\arg \left[G(z)\left(z-z_{1}\right)^{2}\right],\right.
$$

will hold for all interior points on the segment $\left(z_{1}, z_{3}\right)$, where $k$ is some integer".

Princeton University

* See Lemma III of the paper to which reference has already been made, and also Transactions of this Society, vol. 23 (1922), pp. 67-88, Theorem II. 